\title{
Nathalie BAjos \& Michel Bozon (dir.), Enquête sur la sexualité en France. Pratiques, genre et santé
}

Paris, La Découverte, 2008, 609 p.

\section{Sylvie Chaperon}

\section{CpenEdition}

Journals

Édition électronique

URL : http://journals.openedition.org/clio/9780

DOI : $10.4000 /$ clio.9780

ISSN : 1777-5299

Éditeur

Belin

Édition imprimée

Date de publication : 1 mai 2010

Pagination : 324-326

ISSN : $1252-7017$

Référence électronique

Sylvie Chaperon, « Nathalie Basos \& Michel Bozon (dir.), Enquête sur la sexualité en France. Pratiques, genre et santé », Clio. Femmes, Genre, Histoire [En ligne], 31 | 2010, mis en ligne le 21 juin 2010, consulté le 21 septembre 2020. URL : http://journals.openedition.org/clio/9780 ; DOI : https://doi.org/10.4000/ clio.9780

Ce document a été généré automatiquement le 21 septembre 2020.

Tous droits réservés 


\section{Nathalie BAJOS \& Michel BOzoN (dir.), Enquête sur la sexualité en France. Pratiques, genre et santé}

Paris, La Découverte, 2008, 609 p.

Sylvie Chaperon

\section{RÉFÉRENCE}

Nathalie BAjOs \& Michel BOzon (dir.), Enquête sur la sexualité en France. Pratiques, genre et santé, Paris, La Découverte, 2008, 609 p.

1 Ce gros livre est le fruit d'un travail collectif, réalisé à l'initiative de l'Agence nationale de recherche sur le sida et les hépatites virales (ANRS), par un collectif de treize chercheur-e-s (sociologues, démographes, épidémiologistes, psycho-sociologues, économistes). Un long questionnaire a été élaboré et soumis par téléphone par soixante et un enquêteurs de l'Institut de sondage Ipsos à plus de 12000 personnes âgées de 18 à 69 ans entre septembre 2005 et mars 2006. Cette enquête dite «Contexte de la sexualité en France » (CSF) est la troisième enquête quantitative sur les comportements sexuels des français après celle dirigée en 1970 par Pierre Simon (2625 personnes interrogées) et l'enquête ACSF (Analyse des comportements sexuels en France, 1992) qui portait sur 20000 personnes.

2 Outre l'analyse de l'évolution des comportements visibles d'un sondage à l'autre, l'enquête CSF répondait à une hypothèse de recherche forte: les contextes de prévention doivent se diversifier en fonction de l'individualisation des normes sexuelles, des conditions de vie matérielle et des rapports de pouvoir entre les hommes et les femmes. Compte tenu de la richesse de l'ensemble, c'est surtout sur ce dernier aspect, qui articule genre et sexualité, que portera cette lecture.

Le rapprochement des comportements sexuels entre les femmes et les hommes est observé depuis longtemps, l'âge au premier rapport sexuel est quasiment le même dans 
les jeunes générations (17,2 ans pour les hommes et 17,6 pour les femmes) et les pratiques sexuelles se diversifient chez les deux sexes même si des écarts persistent. L'orgasme est devenu une norme pour les deux sexes $(90 \%$ des hommes et $76 \%$ des femmes ont dit l'avoir atteint lors de leur dernier rapport). Si les cunnilingus et fellation sont devenus des pratiques courantes dans les deux sexes, une nette différence se maintient quant à la masturbation que $90 \%$ des hommes disent avoir déjà pratiquée contre $60 \%$ des femmes. De même, le recours à la prostitution ne diminue pas : après 50 ans plus d'un homme sur quatre y a eu recours. On le sait, les hommes déclarent en moyenne beaucoup plus de partenaires que les femmes (11,6 contre 4,4), bien que cet écart se réduise chez les plus jeunes. Plusieurs hypothèses ont été testées et écartées pour expliquer ce fossé persistant: les partenaires prostituées ne peuvent expliquer qu'une partie de la différence; les femmes n'omettent pas plus que les hommes de compter leurs partenaires « sans importance ». Il faut donc en conclure à une définition très genrée de la notion même de partenaire.

Les chercheurs mettent en avant « une double asymétrie [qui] persiste dans la manière de percevoir la vie sexuelle et affective, où semblent toujours s'opposer un désir et des besoins "quasi physiologiques" masculins et des "aspirations affectives et une disponibilité féminines»(p.359). C'est ainsi que les femmes sont quatre fois plus nombreuses que les hommes à confier accepter des rapports dont elles n'ont pas envie pour faire plaisir à leur partenaire, tandis que les hommes sont deux fois plus nombreux à dire qu'ils n'ont jamais rencontré cette situation. Le souhait d'une activité sexuelle plus fréquente est très élevé pour les deux sexes au début d'une relation, mais plus le couple est ancien, plus le désir s'érode, surtout chez les femmes. Ce déclin du désir féminin est plus marqué quand la relation de couple est inégalitaire quant aux tâches domestiques et à la prise en charge des enfants, ce qui représente le cas général. Les hommes eux, se montrent plus affectés par les difficultés financières ou la perte d'emploi.

5 Les violences sexuelles (conversations à caractère pornographique, attouchements, rapports forcés) sont trois fois plus souvent rapportées par les femmes que les hommes. C'est presque toujours un homme qui agresse, même quand la victime est masculine. Par rapport aux enquêtes précédentes et notamment l'Enquête nationale sur les violences à l'encontre des femmes (2000), les déclarations de rapports sexuels forcés sont en nette progression : $7.3 \%$ des femmes ( $2,1 \%$ des hommes) déclarent avoir subi au moins un rapport sexuel forcé, elles n'étaient que 2,7 en 2000. Ceci signale non pas une augmentation de ces violences mais une modification du seuil social de tolérance à leur endroit du fait des débats publics croissants sur le sujet. Pour autant la judiciarisation de ces actes reste minime. Ainsi 0,3\% des femmes (soit entre 36000 et 90000 femmes) ont déclaré avoir subi un rapport sexuel forcé au cours des douze derniers mois, mais moins de 10000 plaintes pour viol ont été déposées la même année. Néanmoins, ces violences ont été révélées par les victimes à une tierce personne dans la moitié des cas, cette proportion augmentant chez les jeunes, surtout quand les violences ont été subies dans l'enfance. En revanche, les agressions subies par les femmes adultes du fait de leur conjoint ou partenaire, et les violences subies par les hommes, quel qu'en soit le contexte, demeurent peu dicibles. Il semblerait donc que les violences sexuelles soient amenées à être de plus en plus déclarées, du moins si les campagnes de prévention continuent et ciblent les victimes encore trop oubliées. 
6 L'enquête CSF renoue avec la perspective proposée par Kinsey en invitant les personnes interrogées à situer leur sexualité sur un gradient allant de l'hétérosexualité exclusive à l'homosexualité. $4 \%$ des hommes comme des femmes déclarent avoir déjà eu des pratiques sexuelles avec un partenaire du même sexe, ce qui témoigne d'une nette augmentation des déclarations féminines par rapport à l'enquête ACSF. Les taux augmentent chez les jeunes et les diplômés. Les personnes qui ont déclaré avoir eu des pratiques homosexuelles dans les douze derniers mois ont des répertoires sexuels plus diversifiés que les autres : elles ont débuté leur vie sexuelle plus jeunes, ont davantage de partenaires sexuels, ont une fréquence plus élevée de rapports sexuels et des pratiques plus diversifiées, elles vivent également moins souvent en couple. La tolérance sociale à l'égard de l'homo ou de la bisexualité s'accrô̂t avec le niveau d'études et reste, quel que soit l'âge, plus marquée chez les femmes que chez les hommes. En revanche l'acceptation de l'homoparentalité, surtout masculine, demeure encore minoritaire.

7 Bien d'autres lectures de cette enquête très riche sont possibles, notamment pour qui s'intéresse à l'impact des inégalités sociales ou aux questions de santé publique. Bien que s'appuyant fortement sur des chiffres et des taux, le texte est rendu parfaitement lisible par le regroupement des statistiques en tableaux et par la mise en encadré des questions techniques complexes. La multiplication des angles d'approches et des questionnements découpe l'ouvrage en vingt-six chapitres qui sont autant d'invitations à l'exploration. 